\title{
Inferred acoustic rates of solar $p$ modes from several helioseismic instruments
}

\author{
F. Baudin ${ }^{1}$, R. Samadi2,3 ${ }^{2,}$ M.-J. Goupil ${ }^{2}$, T. Appourchaux ${ }^{4,1}$, C. Barban ${ }^{5, \star}$, \\ P. Boumier ${ }^{1}$, W. J. Chaplin ${ }^{6}$, and P. Gouttebroze ${ }^{1}$ \\ 1 Institut d'Astrophysique Spatiale, CNRS/Université Paris XI UMR 8617, 91405 Orsay Cedex, France \\ 4 European Space Agency, Research and Science Support Department, Science Payload and Advanced Concept Office, \\ ${ }^{6}$ School of Physics and Astronomy, University of Birmingham, Edgbaston, Birmingham B15 2TT, UK
} e-mail: frederic.baudin@ias.u-psud.fr

2 Observatoire de Paris, LESIA, CNRS UMR 8109, 92195 Meudon, France

3 Observatório Astronómico UC, FCTUC, Coimbra, Portugal PO Box 299, 2200Ag Noordwijk, The Netherlands

5 National Solar Observatory, 950 N. Cherry Avenue, Tucson AZ 85719, USA

Received 4 May 2004 / Accepted 13 November 2004

\begin{abstract}
Acoustic rates of excitation of solar $p$ modes can be estimated from observations in order to place constraints on the modelling of the excitation process and the layers where it occurs in the star. For several reasons (including a poor signal to noise ratio and mode overlap), this estimation is difficult. In this work, we use three completely independent datasets to obtain robust estimates in the solar case for $\ell=1$ modes. We also show that the height in the solar atmosphere where the modes are observed must be taken into account. Our three sets of results are shown to be consistent, particularly in the lower part of the $p$-mode spectrum (from $1.8 \mathrm{mHz}$ to $2.8 \mathrm{mHz}$ ). At higher frequencies, the agreement is not as good, because of a larger dispersion of the measurements and also because of some systematic differences which might be due to observation height estimation or to a systematic influence of the noise.
\end{abstract}

Key words. Sun: oscillation - Sun: helioseismology

\section{Introduction}

Solar seismology has been used for many years to "sound out" the interior of the Sun through information provided by the acoustic resonances (the so-called $p$ modes). More precisely, the frequencies of these resonances - which are usually extracted by the analysis of power frequency spectra are governed by the physical conditions inside the Sun, and these can be "inverted" to infer these conditions (e.g., sound speed, density, rotation). In the last ten years, new ways of analysing the solar oscillations or extracting information from them have been developed, mainly in the field of local seismology (analysing local propagation of acoustic waves). However, globally coherent, or "classical" seismology, is also developing and giving rise to new results: for example Houdek et al. (2001) have used the width of the resonances to infer convection properties. Nevertheless, the outer layers of the Sun remain poorly described, as well as the convection in these layers.

Seismology can place constraints on the modelling of these layers, and on the convection which excites the acoustic resonances. Among others, Samadi \& Goupil (2001) have

^ Present address: Instituut voor Sterrenkunde, Katholieke Universiteit Leuven, Celestijnenlaan 200 B, 3001 Leuven, Belgium. proposed such a model and compared the acoustic rates thereby predicted (see Sect. 2) with observations (Samadi et al. 2003). They found a good overall agreement when the input data of the theoretical model of stochastic excitation were constrained with a 3-D simulation of the Sun. However, even if the agreement is good for the lower part of the $p$-mode spectrum, some clear discrepancies remain at higher frequencies, which is unfortunately where the properties are most difficult to measure.

The excitation rate of low-frequency $p$ modes is mainly dominated by inertia. In contrast, the excitation of highfrequency $p$ modes is more sensitive to the nature of the source of excitation (either dynamical via Reynolds stresses or thermal via turbulent entropy fluctuations, see Goldreich et al. 1994; Samadi et al. 2001). The excitation rate at high frequencies also depends on the temporal properties of the turbulence in the outer layers of the star (Samadi et al. 2003). Observational constraints are needed for the modelling of such properties.

The estimation of the power and width of the acoustic resonances is subject to several sources of error. First, there is a large uncertainty in the measurement as the power spectrum of a resonance (or mode) has a large variance because of the stochastic nature of the excitation (see Sect. 6). Moreover, the absolute calibration in amplitude of a Doppler time 
series is a complex problem (see Sect. 4). Another problem is to know exactly at which height in the solar atmosphere the modes are observed. Oscillations of the photosphere are measured in two ways: photometry in a given wavelength range, or Doppler measurements on a line formed in the photosphere (like the data used in this work). As the density drops rapidly with height in the solar atmosphere, the observed amplitude increases rapidly. Thus, a measurement of power is meaningful only if the altitude to which it corresponds is known. With a proper calibration, comparisons of the absolute power levels of models of mode excitation by convection are possible (whose results are strongly dependent on height; see Sect. 5).

Our aim in this paper is to obtain reliable measurements of the excitation rate of globally coherent, low-angular degree (or low $-\ell$ ) modes. To have confidence in the results we have used three independent datasets (see Sect. 3) covering the same observation period, and then analysed these with the same method and software. We have been particularly careful in our extraction of the parameters at high frequencies (see Sect. 6). Furthermore, we have also taken into account in our analysis the observation heights in the solar atmosphere at which the three datasets were "taken". As such, we have attempted to make available to modellers an internally consistent set of results.

\section{Assessing excitation rates from seismic measurements}

\subsection{Relations between the mode excitation rate, line-width and surface velocity}

The rate at which a $p$ mode of frequency $v_{0}$ is excited is expressed as in Goldreich et al. (1994):

$P=2 \pi \Gamma E$

where $\Gamma=2 \eta / 2 \pi$ is the linewidth of the mode and $\eta$ is the damping rate. The averaged mode energy, $E$, is given by Samadi et al. (2001)

$E=\mathcal{M}\left(r_{\mathrm{s}}\right) v^{2}\left(v_{0}\right)$,

where $v^{2}\left(v_{0}\right)$ is the mean-square surface velocity of a radial mode. The mode mass is obtained from

$\mathcal{M}\left(r_{\mathrm{s}}\right)=\frac{I}{\xi_{\mathrm{r}}^{2}\left(r_{\mathrm{s}}\right)}$ with $I \equiv \int_{0}^{M_{\odot}} \mathrm{d} m \boldsymbol{\xi}^{*} \cdot \boldsymbol{\xi}$

where $I$ is the mode inertia, $\boldsymbol{\xi}$ is the eigenfunction for the fluid displacement, $\xi_{\mathrm{r}}$ its radial component and $r_{\mathrm{s}}$ is the radius at which oscillations are measured.

According to Eqs. (1) and (2), the excitation rate $P$ can then be related to the seismic data by:

$P\left(v_{0}\right)=2 \pi \Gamma \mathcal{M} v^{2}\left(v_{0}\right)$,

where the quantities $\Gamma$ and $v^{2}$ are indeed obtained from the observations.

A pulsation code is used to compute the eigenfunctions, $\xi$, and the mode mass $\mathcal{M}$ for a calibrated solar model (see Sect. 2.3). It is important to stress that the radius $r_{\mathrm{s}}$, at which $\mathcal{M}$ must be evaluated depends on the spectral line of the corresponding seismic measurement (see Sect. 5) and therefore on the observation set and instrument.

\subsection{Determination of the mode surface velocity and line-width from the seismic measurements}

To a first approximation, the mode profile in the observed power velocity spectrum, $\mathcal{P}_{\mathrm{L}}$, can reasonably be assumed to be Lorentzian with a maximum power spectral density, or height, $\mathcal{H}$, and a linewidth at half maximum given by $\Gamma$. The mode profile is then written as:

$\mathcal{P}_{\mathrm{L}}(x)=\mathcal{H} \frac{1}{1+x^{2}}$ where $x \equiv 2\left(v-v_{0}\right) / \Gamma$

The square of the mode velocity, $v^{2}-$ which will be used in evaluating Eq. (4) - results from the integration of the mode profile over the frequency $v$ :

$v_{L}^{2}=\int_{-\infty}^{+\infty} \mathrm{d} v \mathcal{P}_{\mathrm{L}}(v)$

The integration is performed over $]-\infty,+\infty$ [ to take into account both the negative and the positive side of the spectrum. In practice, the real velocity profile is modified by several effects such as the observational technique and geometrical effects. This is taken into account through a multiplicative factor $C_{\mathrm{obs}}$, so that finally one has:

$v_{\mathrm{L}}^{2}=\pi \mathcal{H} \Gamma C_{\mathrm{obs}}$

In the actual observed velocity spectrum the solar $p$ modes exhibit asymmetric profiles. This asymmetry is interpreted as due to the interaction between the resonant cavity mode and local emission from discrete sources (Duvall et al. 1993; Abrams \& Kumar 1996). Nigam et al. (1998) developed a more sophisticated model by adding a correlation between the mode oscillation and the solar noise. After dropping an additional parameter, whose influence applies only far in the wings of the peak and which cannot be observed in the solar case because of the presence of noise and neighbour modes, and after some simplifying approximations, they modelled the power velocity spectrum as (hereafter Nigam's profile):

$\mathcal{P}_{\mathrm{N}}(x)=\mathcal{H} \frac{(1+B x)^{2}+B^{2}}{1+x^{2}}$,

where $B$ is a parameter which controls the asymmetry and contains the effects of correlated noise and of the source. The corresponding mean square velocity of the Nigam profile is the integral of Eq. (8) over $x=]-\infty,+\infty$ [. However, since the approximation performed by Nigam et al. (1998) in deriving Eq. (8) is valid only for $|B x| \ll 1$, the formalism cannot be used when $|x| \gg 1$. In what follows, we obtain estimates of the $\mathcal{H}$ and $\Gamma$ by fitting the Nigam profile (Eq. (8)) to the data. However, we do so by restricting the fitting interval, which runs from $-x_{0}$ to $x_{0}$, to $x_{0} \leq 10$. 
Once the quantities $\mathcal{H}$ and $\Gamma$ are determined, one needs to compute the velocity. The mean square velocity of the mode, $v_{\mathrm{N}}^{2}$, is then:

$v_{\mathrm{N}}^{2}=\pi \mathcal{H} \Gamma C_{\text {obs }}(1+\delta(B))=v_{\mathrm{L}}^{2}(1+\delta(B))$,

with

$\delta(B)=-1+\frac{1}{\pi} \int_{-x_{0}}^{+x_{0}} \mathrm{~d} x \frac{(1+B x)^{2}+B^{2}}{1+x^{2}}$.

The quantity $\delta$ depends on $B$, which in turn depends on $v_{0}$, and is found to be negligible for all the modes investigated here. For instance, at $v_{0} \simeq 3.3 \mathrm{mHz}$ and with $x_{0} \equiv 10$ (i.e., $\left|v-v_{0}\right|=5 \Gamma$ ) we have $B \sim-10^{-2}$ and $\delta \sim 0.05 \%$. This last value must be compared with the relative errors associated with $v^{2}$ which are $\gtrsim 20 \%$. Therefore by estimating $v^{2}$ from Eq. (9) but neglecting the $\delta(B)$ we introduce a negligible error in the determination.

\subsection{Mode mass calculation}

The solar model we consider is calculated with the CESAM code (Morel 1997) and appropriate input physics, as described in detail in Lebreton et al. (1999). In particular, convection is modelled according to the classical mixing-length theory (Böhm-Vitense 1958, hereafter MLT) with a mixing-length $l=\alpha_{\mathrm{c}} H_{\mathrm{p}}$, where $H_{\mathrm{p}}$ is the pressure scale height and $\alpha_{\mathrm{c}}$ is the mixing-length parameter. In contrast with Lebreton et al. (1999), the atmosphere is calculated assuming the Eddington classical gray atmosphere, and microscopic diffusion is included according to the simplified formalism of Michaud \& Proffitt (1993). The calibration of the solar model in luminosity and radius for an age of $4.65 \mathrm{Gyr}$ fixes the initial helium content $Y=0.2751$, the metallicity $Z=0.0196$ and the MLT parameter $\alpha_{\mathrm{c}}=1.76$.

The oscillation eigenfunctions, and hence the mode masses, $\mathcal{M}$, in Eq. (4) are next obtained with the adiabatic pulsation code of Tran Minh \& Leon (1995) from the solar model.

In order to measure the influence of the treatment of convection and of the atmosphere, we have considered two additional solar models. One solar model assumes Canuto et al. (1996)'s local treatment of convection. The second has its atmosphere calculated assuming Kurucz's model, computed such as to provide a good agreement between synthetic and observed Balmer line profiles.

At fixed $r_{\mathrm{s}}$, we find that the mode masses depend on the treatment of convection and on the treatment of the atmosphere but the associated changes in $\mathcal{M}(v)$ are found to be of the same order as the observational error bars associated with the excitation rates. As we are, here, mainly interested in the comparison between observations from different instruments, the effect of the adopted physics on $\mathcal{M}$ does not influence the results.

\section{The different data sets used}

Three datasets were used in this work, each covering the 805-d period from 1996 April to 1998 June. This epoch coincides with a period of low activity on the Sun. The use of several sets allows a direct comparison, and an assessment of the influence on the results of each observation method. The three time series come from the instrument GOLF (Global Oscillation at Low Frequencies, Gabriel et al. 1997) onboard the satellite SoHO, and from the ground-based networks BiSON (Birmingham Solar Oscillation Network, Chaplin et al. 1996) and GONG (Global Oscillation Network Group, Harvey et al. 1996). Each are velocity measurements integrated over the whole solar disc ${ }^{1}$ sensitive to low-degree modes (mainly $\ell=0,1$ and 2). The analysis has been performed in the same way for the three sets (i.e., the same fitting algorithm).

An obvious difference between the datasets is the "duty cycle", or fraction of effective time for which observations are available. Interruptions can result from instrumental failures, and also from bad weather for the ground-based networks. Power levels in the Fourier (frequency) spectrum can be corrected to take account of these interruptions through a simple multiplication by the inverse of the duty cycle. However, as shown by Chaplin et al. (2003), this simple correction does not completely remove the bias in the fitted width and height parameters that is caused by the window. In this work, acoustic rates computed from BiSON and GONG spectra were therefore additionally corrected by a constant value of $9 \%$ for each $10 \%$ change in duty cycle, as indicated by Chaplin et al. (2003).

In addition to this, two aspects play an important role in the comparison of the three datasets. First, the absolute calibration of a Doppler time series is a delicate task. Among the seismic observables, frequencies are very accurately estimated (to some ppm), whereas amplitudes are not. One of the reasons for this is the difficulty in properly deriving the photospheric displacement from the spectro-photometric measurements performed by the instruments. The case of GOLF can be cited as a good example as, because of technical problems, Doppler shifts are estimated from only one wing of the selected atomic lines. However, the task is not much easier for the other two instruments (Hill, Leibacher, private communication). This is detailed in Sect. 4.

The second aspect of the problem of acoustic rate estimation is the height in the solar photosphere to which the observations correspond, as the amplitude of the modes varies with height. This is the old and complex problem of line formation in the solar atmosphere, illustrated by an abundant literature, and detailed in Sect. 5.

\subsection{GOLF data}

GOLF is based on a non-imaging spectrophotometer using the $\mathrm{NaI} \mathrm{D}_{1}$ (at $589.6 \mathrm{~nm}$ ) and $\mathrm{D}_{2}$ lines (at $589.0 \mathrm{~nm}$ ) to measure the velocity of the photosphere. Because of technical problems (Gabriel et al. 1997), the velocity is derived from intensity measurements in only one wing of the lines (the blue wing for the period used here). The average position of the measurement points in the wing is $108 \mathrm{~m} \AA$ from the centre for the $\mathrm{D}_{1}$ line, and 81 and $135 \mathrm{~m} \AA$ for the $\mathrm{D}_{2}$ lines. To this must be added the value of the gravitational redshift for the sodium lines: $12.5 \mathrm{~m} \AA$.

${ }^{1}$ GONG also provides imaged observations, but here we have used the whole disc data. 
As GOLF is space-borne, the interruptions in the observations are very few, and the duty cycle reaches almost $100 \%$ over the total duration of the period of observation chosen here.

\subsection{BiSON data}

The BiSON instruments are also non-imaging spectrophotometers, but they work in the KI line at $769.9 \mathrm{~nm}$. The intensity is measured in both wings, at typically $\pm 63 \mathrm{~m} \AA$ from the centre of the line (which presents a gravitational redshift of $16 \mathrm{~m} \AA$ ).

Despite the use of six active sites spread widely in longitude, the network experiences some interruptions yielding a duty cycle of $\approx 74 \%$ for the period used here.

\subsection{GONG data}

The GONG instrument is an imaging Fourier tachometer using $\mathrm{Ni}$ I at $676.8 \mathrm{~nm}$, which measures velocity from the whole line, as described by Jones (1989). The time series used here is the $\ell=0$ dataset (integration over $95 \%$ of the solar disc). Again, there are some gaps in network coverage giving a duty cycle for the period covered of about $\approx 82 \%$.

\section{Data calibration}

The sensitivity of each instrument is not exactly the same: first, the geometrical visibility of the modes must be taken into account to compare those of different degree $\ell$. The limb-darkening influences this parameter for the GOLF and BiSON spectrophotometers (see Appendix in Appourchaux et al. 2000; Christensen-Dalsgaard \& Gough 1982), making it different for each instrument despite the fact that the geometry is intrinsic to the modes. In addition, GOLF and BiSON have their sensitivity influenced by the relative velocity of the instrument (due to the SoHO orbit for GOLF and to Earth orbit and rotation for BiSON, and to the gravitational redshift for both) and also by the solar rotation, as described by ChristensenDalsgaard (1989). The impact of these effects on visibility gives rise to the so-called "Doppler imaging". This yields a $C_{\text {obs }}$ parameter (see Eq. (7)) for each spectrophotometer, and for each degree $\ell$. Based on Appourchaux et al. (2000) and Christensen-Dalsgaard (1989) and on the limb-darkening description of Allen (1991), we have derived this parameter for GOLF and BiSON.

GONG is not a spectrophotometer but a Fourier tachometer. We have derived $C_{\mathrm{obs}}$ for the modes $\ell=1$ and 2 from the value for $\ell=0$ modes (given by F. Hill, private communication) and from their geometrical visibility.

All these values are listed in Table 1.

\section{Observation heights}

As mentioned in Sect. 2.1, the mass of the modes is one of the parameters needed to compute the excitation rate. As this mode mass is the mode inertia (an intrinsic characteristic) normalised by the displacement at the height where the wave is observed, this height must be properly estimated to derive correct values of the excitation rate. However, obtaining reliable formation
Table 1. Observational correction $C_{\mathrm{obs}}$ for the different instruments and modes (see Sect. 4).

\begin{tabular}{cccc}
\hline \hline & $\ell=0$ & $\ell=1$ & $\ell=2$ \\
\hline GOLF & 2.33 & 2.59 & 6.19 \\
BiSON & 2.99 & 3.38 & 8.41 \\
GONG & 3.27 & 3.88 & 10.91 \\
\hline
\end{tabular}

heights of solar lines is a complex problem. In addition, each instrument analyses a well delimited part of the profile of the line, and as from the center of the line to the wings the height of formation varies by hundreds of $\mathrm{km}$, the characteristics of each instrument must be taken into account. For the three relevant spectral lines used here, we have made use of Jones (1989) for GONG, and Bruls \& Rutten (1992) for BiSON and GOLF. The work done by Jones is perfectly suited as it takes into account the manner in which GONG measurements are performed. On the other hand, the work of Bruls \& Rutten is more general, but provides enough information to estimate the formation height of the $\mathrm{K}$ line of BiSON and the $\mathrm{Na}_{1}$ of GOLF. Unfortunately, this work does not include the neighbouring $\mathrm{Na}_{2}$ line.

We have taken these estimates as a first approach and are aware of their limitations. In addition to the missing $\mathrm{D}_{2}$ line, some other aspects are neglected, e.g., integration over the entire solar disc and limb darkening effect. A complete and coherent treatment of the three lines, applied to the specific case of each instrument, is necessary but remains the next step for a future work.

It is not easy to define a formation height since physically it is not a single altitude in the solar atmosphere, but a range of contributing altitudes to the radiation of interest. The heights we extract from the literature must be considered only as estimates of the maximum of contribution. From Fig. 3 of Jones (1989), we have estimated the observation height of GONG at $h_{\mathrm{GONG}} \approx 240 \mathrm{~km}$ above the photosphere. Knowing the average position at which BiSON and GOLF measurements are made in the wings of $\mathrm{K}$ and $\mathrm{Na}$ lines, we have set $h_{\mathrm{BiSON}} \approx 280 \mathrm{~km}$ from Fig. 10 of Bruls \& Rutten (1992), and $h_{\mathrm{GOLF}} \approx 340 \mathrm{~km}$ from their Fig. 11. Again, these estimates have to be taken as approximate, not only for the reasons already mentioned but also because they result from an average of different physical cases (the "hot" and "cool" models of Bruls \& Rutten). More recently, Georgobiani et al. (2003) also showed that the definition of height is not trivial when comparing geometrical height and optical height.

The effect of height formation influences the determined acoustic rates, via the mode mass, mainly at high frequencies $(v \geq 3 \mathrm{mHz})$; the effect is much weaker at lower frequencies (see Fig. 1). As such, this makes the interpretation of the results easier.

\section{Measurements}

The parameters necessary for the derivation of the acoustic rate are listed in Sect. 2. From the observations, two characteristics of the modes are needed: their width and their height in 


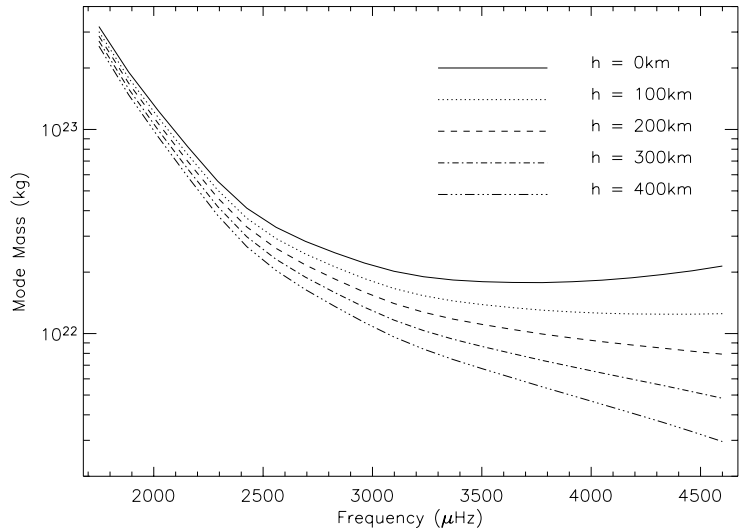

Fig. 1. Mode mass computed for different heights. Because the modes at higher frequencies are concentrated closer to the surface, the masses tend to decrease with increasing frequency. The increasing value of the eigenfunction $\xi$ for larger heights gives the variation of mass versus height.

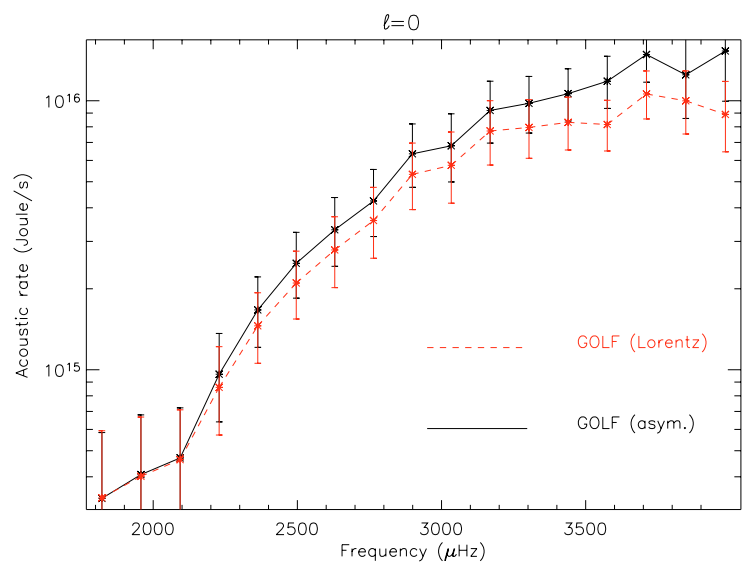

Fig. 2. Excitation rate computed from Lorentzian profiles and asymmetric profiles, showing a significant bias.

the power spectrum. They have been obtained by a classical fitting procedure applied in exactly the same manner to the three data sets. The modes are fitted using the asymmetric profile described by Nigam et al. (1998). This slight deformation of the peak is known to have a significant influence on frequency, but omitting it also leads to a bias in the determination of the acoustic rate, as shown in Fig. 2.

In the frequency interval from 2 to $3.5 \mathrm{mHz}$ in the three datasets, the signal to noise ratio (SNR) is good and the fitting procedure extracts robust estimates of the mode parameters. However, as shown in Samadi et al. (2003), the high frequencies part (higher than $3.5 \mathrm{mHz}$ ) of the spectrum is interesting as observations and models tend to differ here. It is also the most difficult part of the spectrum to analyse, first because of the decreasing SNR, and second because of the increasing width of the modes. This makes the pairs $\ell=0 / 2$ and $\ell=1 / 3$ overlap. This in turn makes the fitting more difficult as cross-talk will appear between the modes.

To minimize the effect of cross-talk between neighbouring modes, as well as between the fitted parameters of a single mode, we modified the fitting strategy at high frequencies. The aim of these changes was to minimize the number of

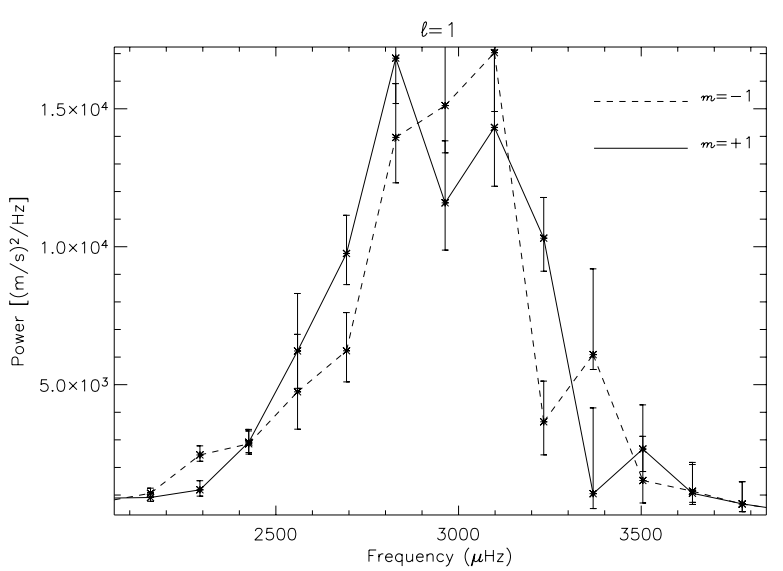

Fig. 3. Fitted heights for $m= \pm 1$ for $\ell=1$ modes, which shows that the hypothesis of equal height for $|m|=\ell$ components is acceptable (error bars are $1 \sigma$ ).

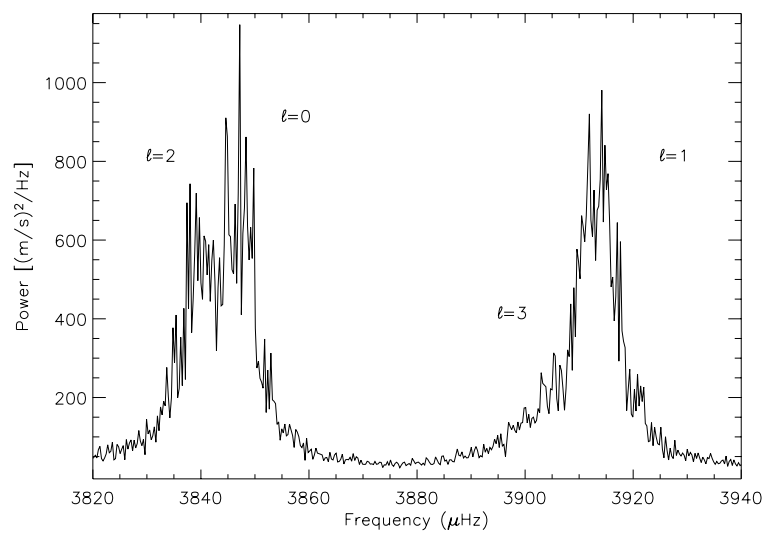

Fig. 4. Power spectrum at high frequencies from GOLF data, showing the overlapping pairs of modes.

fitted parameters; these were tested on the high SNR part of the spectrum and then applied at high frequencies. The changes applied can be something as simple as imposing the same amplitude for the $|m|=\ell$ components of a mode (see Fig. 3), or the same asymmetry for a pair $\ell=0 / 2$ or $\ell=1 / 3$ as shown by Thiery et al. (2000). However, at very high frequencies $(n \geq 29)$, some more parameters have to be fixed in order to avoid poor convergence in the fits. First, we have chosen to restrict ourselves to the extracted $\ell=1$ parameters, as full-disc observations are much less sensitive to $\ell=3$ (see Fig. 4). The comparable heights of the $\ell=0$ and $\ell=2$ make this pair much more difficult to fit. Then, the strategy chosen here consisted of fixing the frequency difference in $\ell=1 / 3$ pairs (leaving the $\ell=1$ frequency to be fitted) in addition to fixing the relative height (from estimates done in the part of the spectrum with good SNR) of the $\ell=3$ mode compared to that of the nearby $\ell=1$ (which is fitted).

An additional way of improving the fitting is to use averaged power spectra. Instead of taking the power spectrum of the whole time series, one divides the series in $N$ subsets, whose $N$ spectra are averaged. It is the averaged spectrum that is then fitted. As the quantity of information is the same in both cases, no improvement should be expected in precision. Nevertheless, as the averaged spectrum has a reduced variance 


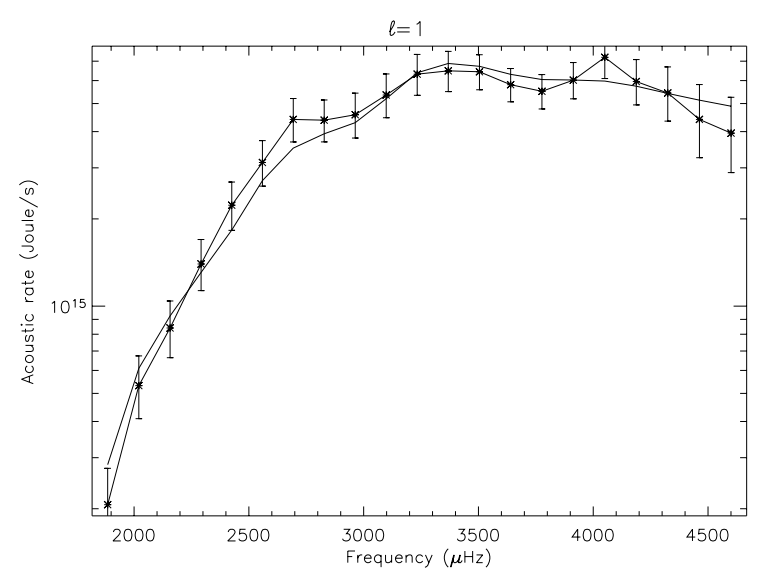

Fig. 5. Comparison of the excitation rate computed from the fitting procedure applied to a simulated spectrum (solid line) and the input excitation rate used for the simulation (crosses).

compared to the single (long) spectrum, and despite a poorer frequency resolution because of the shorter subsets, the fitting algorithm works better at high frequencies where the underlying shape of the peaks in the spectrum becomes then more evident. For this work, we averaged $N=20$ spectra, each made from contiguous 40.25-d pieces. Uncertainties in the fitted parameters were computed according to Appourchaux (2003).

In order to check the reliability of the fitting, we performed a "blind" test using artificial data. The spectrum used is the main Hare-and-Hounds set of the Fitting at Low Angulardegree Group (FLAG) ${ }^{2}$. One of us (WJC) generated the data for the fitter (FB) to analyse, with the basic underlying mode parameters unknown to the latter. As shown in Fig. 5, the output of the fitting procedure are in very good agreement with the input of the simulation, except at very high frequencies where a tendency to a slight underestimation is visible (but generally smaller than the error bars). Even if extensive simulations should be necessary to completely validate the fitting procedure, we consider that this first test is significant.

\section{Results}

\subsection{Raw results}

First, we compare the results for the acoustic rate of excitation of the modes, without any correction for the different observation heights in the solar atmosphere. The mode mass used was computed for an observation height of $h=0 \mathrm{~km}$. This height is certainly not suited for any of the three instruments, but allows a comparison of the raw results in order to check for a possible bias due to the fitting procedure. Corrected results are shown and discussed in Sect. 7.2.

One can see from Fig. 6 that the independent measurements from the three helioseismic instruments coincide reasonably well. The error bars used in Fig. 6 are $1 \sigma$ errors. The different measurements agree typically to within 1 or $2 \sigma$. The agreement between the different datasets is particularly convincing at low frequencies, except for an almost constant difference: in the range $2-3 \mathrm{mHz}$, the GOLF rate is systematically

\footnotetext{
${ }^{2}$ http://bison.ph.bham.ac.uk/ wjc/Research/FLAG.html
}

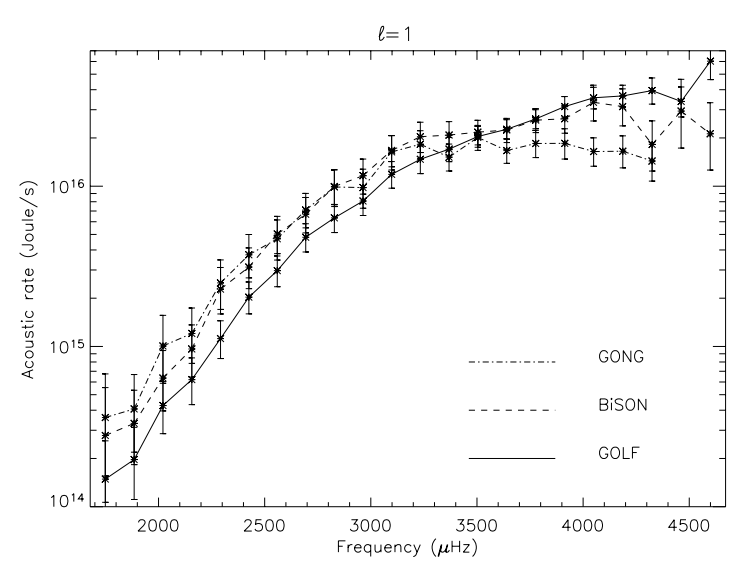

Fig. 6. Raw (no mass mode correction) acoustic rates for the three instruments.

lower than that of BiSON (by about $20 \%$ on average), whereas that of GONG is systematically higher $(\approx 15 \%)$. As mentioned in Sect. 5, mode mass does not make an important difference in this frequency range. The most likely origin of this discrepancy is an inaccurate absolute calibration of the three signals. However, we regard the level of agreement achieved as more than satisfactory given the complexities and uncertainties involved in the calibration.

At higher frequencies ( $v \geq 3 \mathrm{mHz})$, it is the difficulty in fitting the mode parameters (see Sect. 6) that is most problematic. Moreover, the mode mass correction is no longer negligible at these frequencies. However, despite a larger dispersion of the results, the GOLF rates are seen to be sytematically higher than those of BiSON, most of which are in turn higher than those of GONG. This ordering is different from the one seen at low frequencies, and in fact reflects that of the observation heights of the three instruments. It is clearly necessary to correct for this effect.

\subsection{Mode mass corrected results}

The mode masses for the correction applied here were computed using the observation heights chosen in Sect. 5: $h_{\mathrm{GONG}} \approx$ $240 \mathrm{~km}, h_{\mathrm{BiSON}} \approx 280 \mathrm{~km}$ and $h_{\mathrm{GOLF}} \approx 340 \mathrm{~km}$. As expected, the correction does not make a large difference in the comparison of BiSON and GONG, as their observation heights are similar. Also foreseeable was the GOLF results were moved towards those of the other instruments, because of its higher observation height. However, the GOLF results remain higher than the others, particularly if one takes into account the apparent underestimation at low frequencies, which is certainly due to the absolute calibration as discussed in the next section.

\section{Discussion}

A first general conclusion that can be drawn from this work is that the excitation rate of solar $p$ modes can be measured to relatively good accuracy. We have measured it from three completely independent datasets and found that the results were in agreement (without the need for speculative corrections). The discrepancies are of the order of $1 \sigma$ (which corresponds to a 
Table 2. Computed and measured (using GOLF data) parameters for $\ell=1$ modes, including the mode mass correction for acoustic rates.

\begin{tabular}{cccccc}
\hline \hline $\begin{array}{c}\text { Mode } \\
\text { identification }\end{array}$ & $\begin{array}{c}\text { Frequency } \\
(\mu \mathrm{Hz})\end{array}$ & $\begin{array}{c}\text { Mode mass } \\
(\mathrm{kg})\end{array}$ & $\begin{array}{c}\text { Height } \\
(\mathrm{m} / \mathrm{s})^{2} / \mathrm{Hz}\end{array}$ & $\begin{array}{c}\text { Width } \\
(\mu \mathrm{Hz})\end{array}$ & $\begin{array}{c}\text { Supply rate } \\
(\mathrm{Joule} / \mathrm{s})\end{array}$ \\
\hline$n=11 \ell=1$ & 1749.33 & $2.64 \mathrm{e}+23$ & 133.19 & 0.26 & $1.23 \mathrm{e}+14$ \\
$n=12 \ell=1$ & 1885.10 & $1.53 \mathrm{e}+23$ & 256.89 & 0.28 & $1.58 \mathrm{e}+14$ \\
$n=13 \ell=1$ & 2020.83 & $9.58 \mathrm{e}+22$ & 303.58 & 0.47 & $3.32 \mathrm{e}+14$ \\
$n=14 \ell=1$ & 2156.79 & $6.13 \mathrm{e}+22$ & 516.56 & 0.54 & $4.65 \mathrm{e}+14$ \\
$n=15 \ell=1$ & 2292.03 & $4.02 \mathrm{e}+22$ & 717.22 & 0.74 & $8.05 \mathrm{e}+14$ \\
$n=16 \ell=1$ & 2425.57 & $2.84 \mathrm{e}+22$ & 1257.09 & 0.88 & $1.40 \mathrm{e}+15$ \\
$n=17 \ell=1$ & 2559.24 & $2.19 \mathrm{e}+22$ & 1981.39 & 0.94 & $1.96 \mathrm{e}+15$ \\
$n=18 \ell=1$ & 2693.39 & $1.78 \mathrm{e}+22$ & 3890.51 & 0.92 & $3.01 \mathrm{e}+15$ \\
$n=19 \ell=1$ & 2828.15 & $1.48 \mathrm{e}+22$ & 5669.65 & 0.94 & $3.78 \mathrm{e}+15$ \\
$n=20 \ell=1$ & 2963.29 & $1.25 \mathrm{e}+22$ & 11138.21 & 0.80 & $4.55 \mathrm{e}+15$ \\
$n=21 \ell=1$ & 3098.16 & $1.07 \mathrm{e}+22$ & 9901.91 & 1.08 & $6.28 \mathrm{e}+15$ \\
$n=22 \ell=1$ & 3233.13 & $9.44 \mathrm{e}+21$ & 12005.16 & 1.12 & $7.31 \mathrm{e}+15$ \\
$n=23 \ell=1$ & 3368.56 & $8.48 \mathrm{e}+21$ & 5356.12 & 1.84 & $7.90 \mathrm{e}+15$ \\
$n=24 \ell=1$ & 3504.07 & $7.73 \mathrm{e}+21$ & 2781.75 & 2.83 & $8.78 \mathrm{e}+15$ \\
$n=25 \ell=1$ & 3640.39 & $7.07 \mathrm{e}+21$ & 1678.01 & 3.85 & $9.00 \mathrm{e}+15$ \\
$n=26 \ell=1$ & 3776.61 & $6.47 \mathrm{e}+21$ & 831.83 & 5.90 & $9.57 \mathrm{e}+15$ \\
$n=27 \ell=1$ & 3913.49 & $5.95 \mathrm{e}+21$ & 522.33 & 8.09 & $1.04 \mathrm{e}+16$ \\
$n=28 \ell=1$ & 4049.46 & $5.48 \mathrm{e}+21$ & 331.52 & 10.73 & $1.07 \mathrm{e}+16$ \\
$n=29 \ell=1$ & 4186.98 & $5.05 \mathrm{e}+21$ & 236.70 & 12.69 & $9.84 \mathrm{e}+15$ \\
$n=30 \ell=1$ & 4324.79 & $4.64 \mathrm{e}+21$ & 147.81 & 16.39 & $9.42 \mathrm{e}+15$ \\
$n=31 \ell=1$ & 4462.08 & $4.25 \mathrm{e}+21$ & 108.15 & 17.35 & $7.07 \mathrm{e}+15$ \\
$n=32 \ell=1$ & 4599.96 & $3.88 \mathrm{e}+21$ & 78.85 & 26.42 & $1.09 \mathrm{e}+16$ \\
\hline & & & & & \\
& & & & &
\end{tabular}

mean relative error of $22 \%$ for GOLF and $28 \%$ for BiSON and GONG) at low frequencies. These discrepancies can reach a level of 2 or $3 \sigma$ at high frequencies, where the analysis is particularly difficult.

The level of agreement achieved has been possible by using a careful fitting of the data. The model fitted to the observations included an asymmetry in the peaks, since we found that the use of a Lorentzian profile yielded a small but systematic error. A strategy was adopted at high frequencies, where in the fitting of the $\ell=1 / \ell=3$ pairs we used a fixed frequency difference and a fixed amplitude ratio. This gave robust fits despite the poor signal to noise ratio (SNR) and the large width of the peaks. A comparison with simulated data (the Hare-andHounds FLAG spectrum) validated this approach. However, extensive simulations would be necessary to estimate, for example, the influence of a poor SNR at high frequencies.

The agreement between the results from the three sets allows us to put constraints on the excitation rate. The excitation rate can be conveniently expressed as a power law: $P \propto v^{\alpha}$ (see for example Goldreich et al. 1994). At low frequencies, the exponent $\alpha$ mainly represents the inertia dependence of the excitation. At high frequencies, the excitation (and so the slope $\alpha$ ) is strongly dependent on the adopted description of the turbulence. The three sets considered here yield similar values of $\alpha$ at low frequencies $(1.8 \mathrm{mHz} \leq v \leq 2.8 \mathrm{mHz}):(7.7 \pm 0.3)$ for GOLF; $(7.5 \pm 0.4)$ for BiSON; and $(6.9 \pm 0.4)$ for GONG. At higher frequencies $(3.3 \mathrm{mHz} \leq v \leq 4.8 \mathrm{mHz})$, the task is more difficult. First, the raw data (Fig. 6) - which have a positive, null and negative value for $\alpha$-show the necessity of applying

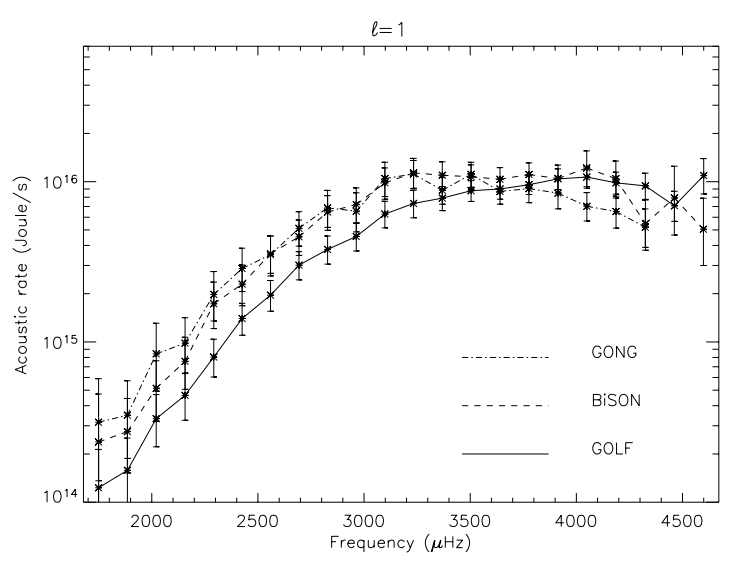

Fig. 7. Mode mass corrected acoustic rates for the three instruments.

a mode mass correction to take account of the height of observation in the solar atmosphere. After the correction has been applied (Fig. 7), the three instruments yield $\alpha$ values of: $(0.3 \pm 0.5)$ for GOLF; $(-2.1 \pm 0.7)$ for BiSON; and $(-2.3 \pm 0.5)$ for GONG. This shows that the mode mass correction brings the GOLF results closer to those of BiSON and GONG.

However, there is still some residual high-frequency discrepancy. This suggests that more work is needed, in particular: (i) on the determination of the observation heights; and (ii) on the influence of the SNR on the measurements at high frequencies. If the Na line used by GOLF were higher than expected in the solar atmosphere, the mode mass correction would be 
stronger and this might possibly make the GOLF results compatible with those of BiSON and GONG. Calculations based on the same hypothesis for radiative transfer in the solar atmosphere for the three atomic lines used are necessary to determine the three heights consistently. Moreover, these calculations must take into account the instrumental details in each case to allow an accurate determination. On the other hand, it must be noted that because of their lower-fill window functions, the SNR in the BiSON and GONG spectra is lower than for GOLF. The poor SNR in the Fourier spectra at high frequencies might bias the observed excitation rate. Extensive numerical simulations are needed to test the extent of this.

This work must be seen as a first step towards a better understanding of $p$-mode excitation and an improved description of convection in the outer layers of the Sun. The use of three different datasets shows that the estimation of the excitation rate is not trivial, as several instrument related effects must be taken into account. However, the results from the three instruments show that it is possible to provide reliable estimates. This is necessary to allow fruitful comparisons between models and observations and to obtain information on the excitation process (is it dominated by Reynolds stress or entropy fluctuation?) and on turbulent convection (for instance its dynamical properties such as the degree of correlation in the turbulent convection). This will be the aim of a following paper.

Acknowledgements. We would like to thank Frank Hill, John Leibacher, and Stuart Jefferies for helpful discussions, Yveline Lebreton for the computation of solar models, and Luca Bertello for providing the calibrated GOLF data. RS's work has been supported by Société de Secours des Amis des Sciences (Paris, France) and by Fundacão para a Ciência e a Tecnologia (Portugal) under grant SFRH/BPD/11663/2002. GOLF is the result of the cooperative endeavours of many individuals, to whom we are deeply indebted. SoHO is a mission of international cooperation between ESA and NASA. We thank all those who have been, or are currently, involved in the BiSON project. BiSON is funded by the UK Particle Physics and Astronomy Research Council (PPARC). This work utilizes data obtained by the Global Oscillation Network Group (GONG) Program, managed by the National Solar Observatory, which is operated by AURA, Inc. under a cooperative agreement with the National Science Foundation. The data were acquired by instruments operated by the Big Bear Solar Observatory, High Altitude Observatory, Learmonth
Solar Observatory, Udaipur Solar Observatory, Instituto de Astrofísica de Canarias, and Cerro Tololo Interamerican Observatory.

\section{References}

Abrams, D., \& Kumar P. 1996, ApJ, 472, 882

Allen, C. W. 1991, Astrophysical Quantities, 3rd ed.

Appourchaux, T. 2003, A\&A, 412, 903

Appourchaux, T., Fröhlich, C., Andersen, B., et al. 2000, ApJ, 538, 401

Böhm-Vitense, E. 1958, Z. Astrophys., 46, 108

Bruls, J. H. M. J., \& Rutten, R. J. 1992, A\&A, 265, 257

Canuto, V. M., Goldman, I., \& Mazzitelli, I. 1996, ApJ, 473, 550

Chaplin, W. J., Elsworth, Y., Howe, R., et al. 1996, Sol. Phys., 168, 1

Chaplin, W. J., Elsworth, Y., Isaak, G. R., et al. 1998, MNRAS, 298, L7

Chaplin, W. J., Elsworth, Y., Isaak, G. R., et al. 2003, A\&A, 398, 305

Christensen-Dalsgaard, J., \& Gough, D. O. 1982, MNRAS, 198, 141

Christensen-Dalsgaard, J. 1989, MNRAS, 239, 977

Duvall, T. L., Jefferies, S. M., Harvey, J. W., Osaki, Y., \& Pomerantz, M. A. 1993, ApJ, 410, 829

Gabriel, A. H., Charra, J., Grec, G., et al. 1997, Sol. Phys., 175, 207

Georgobiani, D., Stein, R. F., Nordlund, Å. 2003, ApJ, 596, 698

Goldreich, P., Murray, N., \& Kumar, P. 1994, ApJ, 424, 466

Harvey, J. W., Hill, F., Hubbard, R., et al. 1996, Science, 272, 1284

Heiter, U., Kupka, F., van't Veer-Menneret, C., et al. 2002, A\&A, 392, 619

Houdek, G., Chaplin, W. J., Appourchaux, T., et al. 2001, MNRAS, 327,483

Jones, H. P. 1989, Sol. Phys., 120, 211

Kurucz, R. L. 1991, in Stellar Atmospheres - Beyond Classical Models, 441

Lebreton, Y., Perrin, M., Cayrel, R., Baglin, A., \& Fernandes, J. 1999, A\&A, 350, 587

Michaud, G., \& Proffitt, C. R. 1993, in Inside the Stars, IAU Coll. 137, ASP Conf. Ser., 40, 246

Morel, P. 1997, A\&AS, 124, 597

Nigam, R., Kosovichev, A. G., Scherrer, P. H., \& Schou, J. 1998, ApJ, 495, L115

Samadi, R., \& Goupil, M.-J. 2001, A\&A, 370, 136

Samadi, R., Goupil, M.-J., \& Lebreton, Y. 2001, A\&A, 370, 147

Samadi, R., Nordlund, Å., Stein, R. F., Goupil, M.-J., \& Roxburgh, I. 2003, A\&A, 404, 1129

Thiery, S., Boumier, P., Gabriel, A. H., et al. 2000, A\&A, 355, 743

Tran Minh, F., \& Leon, L. 1995, in Physical Process in Astrophysics, 219 